\title{
Linear Groups with Restricted Conjugacy Classes
}

\author{
F. de Giovanni ${ }^{1}$ (1) $\cdot$ M. Trombetti ${ }^{1}$ B. A. F. Wehrfritz ${ }^{2}$
}

Received: 3 May 2021 / Accepted: 9 June 2021 / Published online: 21 June 2021

(C) The Author(s) 2021

\section{Abstract}

In this paper we characterize, in terms of their conjugacy classes, linear groups $G$ such that $G / \zeta_{k}(G)$ belongs to a certain group class $\mathfrak{X}$ for several natural choices of $\mathfrak{X}$. Moreover, a description is given of linear groups with restrictions on layers.

Keywords Linear group · Conjugacy class · Layer

Mathematics Subject Classification 20F24 · 20H20

\section{Introduction}

A famous theorem of Schur [11] states that if $G$ is any group whose centre $\zeta_{1}(G)$ has finite index, then its commutator subgroup $G^{\prime}$ is finite. This result was later extended by Baer [1], who proved that the $(k+1)$-th term $\gamma_{k+1}(G)$ of the lower central series of $G$ is finite whenever $G$ is finite over its $k$-th centre $\zeta_{k}(G)$ for some non-negative integer $k$. Although the consideration of an infinite extraspecial group shows that an exact converse of Baer's theorem is false, even for $k=1$, it was shown by Philip Hall [6] that if $G$ is any group with $\gamma_{k+1}(G)$ finite, then also the factor group $G / \zeta_{2 k}(G)$ is finite. On the other hand, Merzljakov proved that for any linear group $G$ the finiteness of $G / \zeta_{k}(G)$ and $\gamma_{k+1}(G)$ are equivalent (see [8] or [13], Corollary 10.20).

The first two authors are supported by GNSAGA (INdAM) and are members of AGTA - Advances in Group Theory and Applications (www.advgrouptheory.com).

$\triangle$ F. de Giovanni

degiovan@unina.it

M. Trombetti

marco.trombetti@unina.it

B. A. F. Wehrfritz

b.a.f.wehrfritz@qmul.ac.uk

1 Dipartimento di Matematica e Applicazioni, Università di Napoli Federico II, Naples, Italy

2 School of Mathematical Sciences, Queen Mary University of London, London, England, UK 
The above results have been generalized by replacing finiteness by other natural finiteness conditions. In particular, it turns out that if $G / \zeta_{1}(G) \in \mathfrak{X}$, then $G^{\prime}$ is an $\mathfrak{X}$-group, whenever $\mathfrak{X}$ is any of the classes $\mathfrak{C}$ of Černikov groups, $\mathfrak{P} \mathfrak{F}$ of polycyclicby-finite groups, $\mathfrak{M}_{\pi}$ of soluble-by-finite $\pi$-minimax groups, $\mathfrak{R}$ of soluble-by-finite groups of finite rank (see the introduction of [5] for more details). Here a group $G$ is said to have finite rank $r$ if every finitely generated subgroup of $G$ can be generated by $r$ elements and if $r$ is the least non-negative integer with this property. Recall also that, if $\pi$ is any set of primes, a soluble-by-finite group $G$ is $\pi$-minimax if it has a series of finite length whose factors are finite or cyclic or of type $p^{\infty}$ for some $p \in \pi$; if $\pi$ is the set of all primes, a $\pi$-minimax group is simply called minimax and the class of minimax groups is denoted by $\mathfrak{M}$. It is known that a soluble-by-finite minimax group is reduced, i.e. it has no radicable non-trivial subgroups, if and only it is residually finite. Notice that Platonov proved that any linear group of finite rank is soluble-by-finite (see [13], Theorem 10.9). Moreover, it has been shown in [5] that a linear group admitting a finite series whose factors satisfy either the minimal or the maximal condition is a soluble-by-finite $\pi$-minimax for some finite set $\pi$ of primes.

Recall that a group $G$ is an $F C$-group if each of its elements has only finitely many conjugates, or equivalently if the centralizer $C_{G}(g)$ has finite index in $G$ for every $g \in G$. Of course, any direct product of finite groups has the $F C$-property, so that there are $F C$-groups with an infinite commutator subgroup and a fortiori whose centre has infinite index. However, it is well known that for a linear group the properties of being $F C$ and central-by-finite are equivalent (see for instance [13], Corollary 5.6). For more details on the theory of $F C$-groups we refer to the monograph [12].

More generally, if $\mathfrak{X}$ is any group class, we say that a group $G$ is an $\mathfrak{X} C$-group, or has $\mathfrak{X}$-conjugacy classes, if the factor group $G / C_{G}\left(\langle g\rangle^{G}\right)$ belongs to $\mathfrak{X}$ for each element $g$ of $G$. In particular, if $\mathfrak{F}$ is the class of all finite groups, a group has $\mathfrak{F}$-conjugacy classes if and only if it is an $F C$-group and so $\mathfrak{X} C$-groups arise as natural generalizations of groups with finite conjugacy classes. If the group class $\mathfrak{X}$ is chosen to be one of the classes $\mathfrak{C}, \mathfrak{P F}$ and $\mathfrak{M}$, we obtain the concepts of $C C$-groups, PC-groups and $M C$-groups, respectively. Groups with the $C C$-property were introduced by Polovickii in [9] and later investigated by several authors, while the study of $P C$-groups was started in [3] and that of $M C$-groups in [7]. Notice that the properties $F C$ and $P C$ obviously coincide for periodic groups, as well as the properties $C C$ and $M C$.

If $\mathfrak{X}$ is any $\mathbf{Q}$-closed group class (i.e. a class of groups which is closed under quotients) and $G$ is a group such that $G / \zeta_{1}(G)$ belongs to $\mathfrak{X}$, then $G$ is an $\mathfrak{X} C$-group. On the other hand, as in the case of $F C$-groups, the imposition of the property $\mathfrak{X} C$ to a group $G$ does not imply that $G / \zeta_{1}(G)$ is in $\mathfrak{X}$, even when $\mathfrak{X}$ is one if the classes $\mathfrak{C}$, $\mathfrak{P F}, \mathfrak{M}$. It is easy to prove that for a linear group the $\mathfrak{X} C$-property is equivalent to the property of being central-by- $\mathfrak{X}$ for the above choices of the group class $\mathfrak{X}$ and the aim of this paper is to further investigate this kind of restrictions on conjugacy classes within the universe of linear groups.

Our notation is mostly standard and can be found in [10]. We refer to [13] for results and terminology concerning linear groups. 


\section{Conjugacy classes}

We start by recalling properties of linear groups that we require. If $G$ is a linear group, denote by $G^{0}$ its connected component containing 1 . It is well known that $G^{0}$ has finite index in $G$ and is contained in every closed subgroup of finite index (see [13], Lemma 5.2 and Lemma 5.3). It follows that $G^{0}$ centralizes each element of $G$ admitting finitely many conjugates, since all centralizers in a linear group are closed (see [13], Lemma 5.4); for the same reason, the centre of a linear group is closed and every linear group satisfies the minimal and the maximal condition on centralizers. Finally, we mention that any soluble connected linear group over an algebraically closed field is triangularizable by the Lie-Kolchin theorem (see [13], Theorem 5.8).

The first lemma of this section proves that in the linear case the $\mathfrak{X} C$-property is equivalent to the property of being central-by- $\mathfrak{X}$ for several natural choices of the group class $\mathfrak{X}$. Recall that a group class $\mathfrak{X}$ is $\mathbf{R}_{0}$-closed if $G / N_{1} \cap N_{2}$ is an $\mathfrak{X}$-group whenever $N_{1}$ and $N_{2}$ are normal subgroups of a group $G$ such that $G / N_{1}$ and $G / N_{2}$ belong to $\mathfrak{X}$.

Lemma 2.1 Let $\mathfrak{X}$ be an $\boldsymbol{R}_{0}$-closed group class and let $G$ a linear $\mathfrak{X} C$-group. Then the factor group $G / \zeta_{1}(G)$ belongs to $\mathfrak{X}$.

Proof Since $G$ satisfies the minimal condition on centralizers, there is a finitely generated subgroup $X$ of $G$ such that $\zeta_{1}(G)=C_{G}(X)$. Then $\zeta_{1}(G)=C_{G}\left(X^{G}\right)$ and hence $G / \zeta_{1}(G)$ is an $\mathfrak{X}$-group because $G$ has the $\mathfrak{X} C$-property and the class $\mathfrak{X}$ is $\mathbf{R}_{0}$-closed.

Lemma 2.2 Let $G$ be a linear group such that $[g, G]$ is soluble-by-finite for each element $g$ of $G$. Then $G$ is soluble-by-finite.

Proof Let $S$ be the maximal soluble normal subgroup of $G$. Then $S$ is closed in $G, \bar{G}=$ $G / S$ is a linear group and $[\bar{g}, \bar{G}]$ is finite for each $\bar{g} \in \bar{G}$. Hence $\bar{G}$ is an $F C$-group, is central-by-finite and so is finite. Therefore $G$ is soluble-by-finite.

Now, a group $G$ has the $C C$-property if and only if for each element $g$ of $G$ both the subgroup $[g, G]$ and the factor group $G / C_{G}([g, G])$ are Černikov; actually, since periodic groups of automorphisms of Černikov groups are likewise Černikov (see [10] Part 1, Theorem 3.29), the condition on $G / C_{G}([g, G])$ may be weakened, requiring only its periodicity. On the other hand, consideration of the holomorph of a Prüfer group proves that in general the assumption on $G / C_{G}([g, G])$ cannot be completely removed. However, this is certainly possible in the case of linear groups, as the following shows.

Lemma 2.3 Let $G$ be a linear group and let $N$ be a Černikov normal subgroup of $G$. Then $G / C_{G}(N)$ is Černikov.

Proof Let $J$ be the largest divisible normal subgroup of $N$ and let $g$ be any element of $G$. Since the connected component $G^{0}$ centralizes $J$ by Lemma 1.12 of [13] and has finite index in $G$, there exists a positive integer $k$ such that $x=g^{k}$ acts trivially on $J$ and $N / J$. It easily follows that $x^{h}$ centralizes $N$ for a suitable $h>0$. Thus $G / C_{G}(N)$ is periodic and hence it is a Černikov group. 
Our next statement provides a characterization of the $C C$-property within the universe of linear groups.

Theorem 2.4 Let $G$ be a linear group. Then the following statements are equivalent:

(a) $G / \zeta_{1}(G)$ is a Černikov group;

(b) $G^{\prime}$ is a Černikov group;

(c) $[g, G]$ is a Černikov group for each element $g$ of $G$;

(d) $G$ is a CC-group.

Proof The implication (a) $\Longrightarrow$ (b) holds for an arbitrary group by a result of Polovickir (see [10], Theorem 4.23). Moreover, (b) $\Longrightarrow(\mathrm{c})$ is obvious and (d) $\Longrightarrow$ (a) is a consequence of Lemma 2.1. Finally, it follows from Lemma 2.3 and the previously quoted characterization of $C C$-groups that a linear group satisfying (c) has the $C C$-property.

The following two consequences of Theorem 2.4 show that linear $C C$-groups behave similarly to Černikov groups; in particular, the second of them provides a characterization of periodic linear $C C$-groups.

Corollary 2.5 Let $G$ be a linear CC-group. Then $G^{0}$ is abelian and $G$ is abelian-byfinite.

Proof Of course, we may suppose that $G$ is linear over an algebraically closed field. Since $G / \zeta_{1}(G)$ is Černikov by Theorem $2.4, G$ is metabelian-by-finite. Hence $G^{0}$ is metabelian and so triangularizable by the Lie-Kolchin theorem. Thus $\left(G^{0}\right)^{\prime}$ is unipotent and hence finite, being Černikov by Theorem 2.4. But $\left(G^{0}\right)^{\prime}$ is connected by Lemma 5.7 of [13], so that $\left(G^{0}\right)^{\prime}=\{1\}$ and $G^{0}$ is abelian.

If $G$ is any periodic group, we use the symbol $\pi(G)$ to denote the set of all primes $p$ such that $G$ has elements of order $p$.

Corollary 2.6 Let $G$ be a periodic linear CC-group over a field of characteristic $q$ and put $\pi=\pi\left(G / \zeta_{1}(G)\right)$.

(a) If $q$ does not belong to $\pi$, then $G=U \times V \times W$, where $U$ is a Černikov $\pi$ subgroup, $V$ is an abelian $q$-subgroup of finite exponent and $W$ is an abelian $(\pi \cup\{q\})^{\prime}$-subgroup of finite rank if $q>0$, while $V=\{1\}$ and $W$ is an abelian $\pi^{\prime}$-subgroup of finite rank if $q=0$.

(b) If $q$ belongs to $\pi$, then $G=U \times W$, where $U=E\left(V_{1} \times V_{2}\right), E$ is a finite $\pi$-subgroup whose order is divisible by $q, V_{1}$ is a central $q$-subgroup of finite exponent, $V_{2}$ is a Černikov $\pi$-subgroup and $W$ is an abelian $\pi^{\prime}$-subgroup of finite rank.

Proof (a) Clearly, the $\pi^{\prime}$-component $A$ of $\zeta_{1}(G)$ is a Hall $\pi^{\prime}$-subgroup of $G$ and so $G=U \times A$ for a suitable $\pi$-subgroup $U$ (see [13], Theorem 9.18). Since $A$ is linear over a field of characteristic $q$, we have $A=V \times W$, where $V$ is an abelian $q$-group of finite exponent and $W$ is an abelian $q^{\prime}$-group of finite rank if $q>0$, while $V=\{1\}$ and $W=A$ has finite rank if $q=0$ (see for instance [13], Theorem 2.2). Finally, for each prime $p \in \pi$ the $p$-component of $U \cap \zeta_{1}(G)$ has finite rank and hence $U$ is a Černikov group (the set $\pi$ is finite by Theorem 2.4). 
(b) Let $J / \zeta_{1}(G)$ be the largest divisible subgroup of the Černikov group $G / \zeta_{1}(G)$. Since the Sylow $q$-subgroups of $G$ have finite exponent, $q$ is not in

$$
\pi_{1}=\pi\left(J / \zeta_{1}(J)\right) \subseteq \pi
$$

and hence by (a) we have $J=U_{1} \times V_{1} \times W_{1}$, where $U_{1}$ is a Černikov $\pi$-subgroup, $V_{1}$ is an abelian $q$-subgroup of finite exponent and $W_{1}$ is an abelian $\left(\pi_{1} \cup\{q\}\right)^{\prime}$-subgroup of finite rank. Write $W_{1}=U_{2} \times W$, where $U_{2}$ is a $\pi$-subgroup and $W$ is a $\pi^{\prime}$-subgroup, so that $W \leq \zeta_{1}(G)$ and $V_{2}=U_{1} \times U_{2}$ is a Černikov $\pi$-subgroup. Clearly there exists a finite $\pi$-subgroup $E$ of $G$ such that $G=E J$. In particular, $q$ divides the order of $E$ and

$$
G=E\left(\left(V_{1} \times V_{2}\right) \times W\right)=U \times W,
$$

where $U=E\left(V_{1} \times V_{2}\right)$ is a $\pi$-subgroup.

The characterization of linear $C C$-groups provided by Theorem 2.4 can actually be generalized to $\mathfrak{X} C$-groups for several other choices of the group class $\mathfrak{X}$. In fact, it can be proved that for a linear group $G$ the following four statements

(a) $G / \zeta_{1}(G) \in \mathfrak{X}$

(b) $G^{\prime} \in \mathfrak{X}$

(c) $[g, G] \in \mathfrak{X}$ for each $g \in G$

(d) $G$ is an $\mathfrak{X} C$-group

are equivalent when $\mathfrak{X}$ is any one of the classes of polycyclic-by-finite, $\pi$-minimax, reduced $\pi$-minimax groups and, provided that the characteristic is positive, the class of groups of finite rank. In fact, it has been remarked in the introduction that (a) $\Longrightarrow$ (b) holds for all these classes, while (b) $\Longrightarrow$ (c) is obvious and (d) $\Longrightarrow$ (a) follows from Lemma 2.1. In order to prove that (d) is a consequence of (c), notice first that the latter condition ensures that the whole group $G$ is soluble-by-finite by Lemma 2.2. Since $\langle g\rangle^{G}=\langle g\rangle[g, G]$ belongs to $\mathfrak{X}$, it follows from Theorem 3.27 of [10] and from Lemma 4.7, Lemma 4.6 and Lemma 3.3 of [5] that $G$ is an $\mathfrak{X} C$-group for all our choices of $\mathfrak{X}$.

Our next aim is to characterize linear groups $G$ such that $G / \zeta_{k}(G)$ belongs to a group class $\mathfrak{X}$ for some integer $k>1$.

Let $\mathfrak{X}$ be any group class. Put $\mathfrak{X} C^{0}=\mathfrak{X}$ and suppose by induction that the group class $\mathfrak{X} C^{k}$ has been defined for some non-negative integer $k$; then we use the symbol $\mathfrak{X} C^{k+1}$ for the class consisting of all groups $G$ such that $G / C_{G}\left(\langle g\rangle^{G}\right)$ belongs to $\mathfrak{X} C^{k}$ for each element $g$ of $G$. Clearly, the properties $\mathfrak{X} C$ and $\mathfrak{X} C^{1}$ coincide. Moreover, if the group class $\mathfrak{X}$ is $\mathbf{Q}$-closed, then $\mathfrak{X} C^{k}$ contains all $\mathfrak{X}$-groups and all groups which are $\mathfrak{X}$ over the $k$-th centre. If we choose as $\mathfrak{X}$ the class $\mathfrak{F}$ of all finite groups, we obtain the class $\mathfrak{F} C^{k}=F C^{k}$ that was introduced and studied in [4], where it was proved that many interesting properties concerning $F C$-groups can be generalized to $F C^{k}$-groups for $k>1$. It turns out for instance that if $G$ is any $F C^{k}$-group, the subgroup $\gamma_{k+1}(G)$ is periodic, so that in particular a torsion-free group has the $F C^{k}$-property if and only if it is nilpotent of class at most $k$. 
Our next result is an extension of Lemma 2.1.

Theorem 2.7 Let $\mathfrak{X}$ be an $\boldsymbol{R}_{0}$-closed class of groups and let $G$ be a linear $\mathfrak{X} C^{k}$-group for some non-negative integer $k$. Then $G / \zeta_{k}(G)$ belongs to $\mathfrak{X}$.

Proof The statement is obvious for $k=0$, so suppose $k>0$. Since $G$ satisfies the minimal condition on closed subgroups, there are finitely many elements $g_{1}, \ldots, g_{m}$ of $G$ such that

$$
\bigcap_{i=1}^{m} C_{G}\left(\left\langle g_{i}^{G}\right\rangle\right)=\zeta_{1}(G)
$$

For each $i=1, \ldots, m$, the linear group $G / C_{G}\left(\left\langle g_{i}{ }^{G}\right\rangle\right)$ has the $\mathfrak{X} C^{k-1}$-property and hence by induction on $k$ the group $G / Z_{i}$ belongs to $\mathfrak{X}$, where

$$
Z_{i} / C_{G}\left(\left\langle g_{i}^{G}\right\rangle\right)=\zeta_{k-1}\left(G / C_{G}\left(\left\langle g_{i}^{G}\right\rangle\right)\right)
$$

If $Z$ is the intersection of all $Z_{i}$ 's, we have

$$
\left[Z,,_{k-1} G\right] \leq \bigcap_{i=1}^{m}\left[Z_{i, k-1} G\right] \leq \bigcap_{i=1}^{m} C_{G}\left(\left\langle g_{i}{ }^{G}\right\rangle\right)=\zeta_{1}(G)
$$

and hence $Z=\zeta_{k}(G)$. It follows now from the $\mathbf{R}_{0}$-closure of $\mathfrak{X}$ that $G / \zeta_{k}(G)$ is an $\mathfrak{X}$-group.

Corollary 2.8 Let $\mathfrak{X}$ be a group class which is $\boldsymbol{Q}$ and $\boldsymbol{R}_{0}$-closed. A linear group $G$ is $\mathfrak{X} C^{k}$ for some non-negative integer $k$ if and only if $G / \zeta_{k}(G)$ belongs to $\mathfrak{X}$.

Notice that the above results apply in particular to the classes of finite, Černikov, polycyclic-by-finite, $\pi$-minimax, finite rank groups and hence they provide a further characterization of linear groups $G$ for which $G / \zeta_{k}(G)$ is finite, Černikov, polycyclicby-finite, $\pi$-minimax or of finite rank, respectively. Although the class $\mathfrak{M}_{\pi}^{\star}$ of solubleby-finite reduced $\pi$-minimax groups is not $\mathbf{Q}$-closed (with the obvious exception $\pi=\emptyset$ ), even within the universe of linear groups, we can prove for this class a result similar to Corollary 2.8 in the context of connected linear groups. To this aim, we need the following lemma which holds in particular for soluble connected linear groups by the Lie-Kolchin theorem. The proof of this auxiliary result makes use of the Jordan decomposition. Recall that, if $G$ is a linear group of degree $n$ over an algebraically closed field $\mathfrak{K}$ and $g$ is any element of $G$, then $G L(n, \mathfrak{K})$ contains a unique unipotent element $g_{u}$ and a unique diagonalizable element $g_{d}$ such that $g=g_{u} g_{d}=g_{d} g_{u}$ : this is called the Jordan decomposition of $g$ (see for instance [13], Theorem 7.2). Put

$$
G_{u}=\left\{g_{u} \mid g \in G\right\} \text { and } G_{d}=\left\{g_{d} \mid g \in G\right\}
$$

If $G$ is locally nilpotent, then $G_{u}$ and $G_{d}$ are subgroups of $G L(n, \mathfrak{K})$ and

$$
G \leq\left\langle G_{u}, G_{d}\right\rangle=G_{u} \times G_{d}=G_{u} G=G G_{d}
$$


(see [13], 7.14). Notice that $G_{u}$ is unitriangularizable (see [13], Corollary 1.21) and hence it is either primary or torsion-free, according to the characteristic.

Lemma 2.9 Let $G \leq \operatorname{Tr}(n, \mathfrak{K})$, where $\mathfrak{K}$ is a field and $n$ is a positive integer. Then the largest divisible subgroup of $\zeta_{k+1}(G) / \zeta_{k}(G)$ is torsion-free for every positive integer $k$.

Proof Of course, we may suppose $\mathfrak{K}$ algebraically closed. Let $J / \zeta_{k}(G)$ be the largest periodic divisible subgroup of $\zeta_{k+1}(G) / \zeta_{k}(G)$ and put

$$
G^{*}=G\left(\zeta_{k+1}(G)_{u} \times \zeta_{k+1}(G)_{d}\right)
$$

where $\zeta_{k+1}(G)_{u}$ and $\zeta_{k+1}(G)_{d}$ are respectively the unipotent and the diagonalizable components of $\zeta_{k+1}(G)$ determined by the Jordan decomposition of its elements. Clearly, $G^{*} \leq \operatorname{Tr}(n, \mathfrak{K})$ and $\zeta_{k+1}\left(G^{*}\right)_{d}$ is $G$-invariant. Moreover,

$$
J_{d} \leq \zeta_{k+1}(G)_{d} \leq \zeta_{k+1}\left(G^{*}\right)_{d}
$$

(see [13], Lemma 7.17), so that $\left[J_{d}, G\right]=\{1\}$ and hence $J_{d} \leq \zeta_{1}\left(G^{*}\right) \leq \zeta_{k}\left(G^{*}\right)=Z$.

Then

$$
J Z / Z \leq J_{u} J_{d} Z / Z=J_{u} Z / Z
$$

But $J_{u} Z / Z$ is unipotent and as such is torsion-free or has finite exponent. On the other hand, $\zeta_{k}(G) \leq Z$ again by Lemma 7.17 of [13], so that $J Z / Z$ is divisible periodic and hence trivial. Therefore $J \leq G \cap Z \leq \zeta_{k}(G)$ and the statement is proved.

The above lemma should be seen in relation to the well known fact that the factor group $\zeta_{k+1}(G) / \zeta_{k}(G)$ is reduced for any periodic group $G$ and any positive integer $k$.

Theorem 2.10 Let $\pi$ be a set of primes. If $G$ is a connected linear group and $k$ is a positive integer, then the following statements are equivalent.

(a) $G / \zeta_{k}(G)$ is $\pi$-minimax.

(b) $G / \zeta_{k}(G)$ is reduced $\pi$-minimax.

(c) $G$ is an $\mathfrak{M}_{\pi}^{\star} C^{k}$-group.

Proof Suppose that $G / \zeta_{k}(G)$ is $\pi$-minimax. Since $\zeta_{k}(G)$ is closed, the factor group $G / \zeta_{k}(G)$ is linear and so soluble-by-finite. Then $G$ itself is soluble-by-finite and hence even soluble. Let $J / \zeta_{k}(G)$ denote the finite residual of $G / \zeta_{k}(G)$, so that $G / J$ is reduced $\pi$-minimax. The connected group $G / \zeta_{k}(G)$ centralizes every element with finitely many conjugates, so that

$$
J / \zeta_{k}(G) \leq \zeta_{1}\left(G / \zeta_{k}(G)\right)=\zeta_{k+1}(G) / \zeta_{k}(G)
$$

Application of Lemma 2.9 yields that $J=\zeta_{k}(G)$ and hence $G / \zeta_{k}(G)$ is reduced $\pi$-minimax. It now follows that (a) and (b) are equivalent. 
Now (c) implies (b) by Theorem 2.7 and so we need only to prove that (c) is a consequence of (b). Suppose $G / \zeta_{k}(G)$ is reduced $\pi$-minimax and induct on $k$. If $k=1$ then (c) holds by the comments after Corollary 2.6. Assume $k \geq 2$. Let $g \in G$ and set $C=C_{G}\left(\langle g\rangle^{G}\right)$ and $\bar{G}=G / C$. Obviously, $\zeta_{1}(G) \leq C$, so $\zeta_{k}(G) C / C$ lies in $\zeta_{k-1}(\bar{G})$ and hence $\bar{G} / \zeta_{k-1}(\bar{G})$ is at least $\pi$-minimax. But $C$ is closed in $G$, so $\bar{G}$ is isomorphic to a connected linear group and thence $\bar{G} / \zeta_{k-1}(\bar{G})$ is reduced $\pi$-minimax because (a) and (b) are equivalent. By induction $\bar{G}$ belongs to $\mathfrak{M}_{\pi}^{\star} C^{k-1}$ and therefore $G$ is an $\mathfrak{M}_{\pi}^{\star} C^{k}$-group. The proof is complete.

Corollary 2.11 Let $\pi$ be a set of primes. If $G$ is a linear group with $G / \zeta_{k}(G) \pi$-minimax for some positive integer $k$, then $G$ is a finite extension of an $\mathfrak{M}_{\pi}^{\star} C^{k}$-group.

Proof Since $G^{0} \cap \zeta_{k}(G) \leq \zeta_{k}\left(G^{0}\right)$, the factor group $G^{0} / \zeta_{k}\left(G^{0}\right)$ is $\pi$-minimax and so the connected group $G^{0}$ has the $\mathfrak{M}_{\pi}^{\star} C^{k}$-property by Theorem 2.10 . The statement is proved.

\section{Layers}

In this section we study linear groups with restrictions on their layers. Recall that if $G$ is a group and $k$ is an element of the set $\mathbb{N} \cup\{\infty\}$, the subgroup $G_{k}$ generated by all elements of $G$ of order $k$ is called the $k$-layer of $G$. If $\mathfrak{X}$ is any group class, a group $G$ is called an $\mathfrak{X} L$-group if all its layers belong to $\mathfrak{X}$. Notice that if $G$ is an $\mathfrak{X} L$-group and $g$ is an element of infinite order of $G$, the subgroup $\left\langle g, \zeta_{1}(G)\right\rangle$ is generated by its elements of infinite order and so is contained in the $\infty$-layer $G_{\infty}$; in particular we have the following result.

Lemma 3.1 Let $\mathfrak{X}$ be a subgroup closed group class and let $G$ be a non-periodic $\mathfrak{X} L$-group. Then the centre $\zeta_{1}(G)$ belongs to $\mathfrak{X}$.

Groups with Černikov layers are usually called $C L$-groups. Clearly, any $C L$-group has a covering consisting of Černikov normal subgroups and hence is a periodic $C C$-group. Moreover, it is clear that in a $C L$-group all subgroups of finite exponent are finite and hence the Sylow $p$-subgroups of any group with Černikov layers are Černikov. The following easy consequence of Corollary 2.6 characterizes linear $C L$-groups.

Corollary 3.2 A group $G$ is linear and has the CL-property if and only if $G=A \times C$, where $A$ is a periodic abelian subgroup of finite rank, $C$ is a Černikov subgroup and $\pi(A) \cap \pi(C)=\emptyset$.

Using the above corollary, we may also characterize linear $F$ L-groups, i.e. linear groups with finite layers. In fact, every such group has finite conjugacy classes and in particular every abelian-by-finite $F L$-group is finite over its centre.

Corollary 3.3 A group $G$ is linear and has the FL-property if and only if $G=A \times C$, where $A$ is a periodic abelian group of finite rank, $C$ is a Černikov central-by-finite group and $\pi(A) \cap \pi(C)=\emptyset$. 
The following result shows that the study of linear groups with minimax layers essentially can be reduced to that of $C L$-groups.

Corollary 3.4 Let $\pi$ be a set of primes and let $G$ be a linear group with $\pi$-minimax (reduced $\pi$-minimax) layers. Then either $G$ is $\pi$-minimax (reduced $\pi$-minimax) or it has Černikov layers (finite layers).

Proof For each element $g$ of $G$ the subgroup $[g, G] \leq\langle g\rangle^{G}$ is (reduced) $\pi$-minimax, so that also the linear group $G / \zeta_{1}(G)$ is (reduced) $\pi$-minimax (see the discussion after Corollary 2.6). It follows now from Lemma 3.1 that either $G$ is periodic or $\zeta_{1}(G)$ is (reduced) $\pi$-minimax; in the former case, $G$ has Černikov layers, or even finite if they are reduced. The statement is proved.

If in the above statement we choose $\pi=\emptyset$, we obtain the following characterization of linear groups with polycyclic-by-finite layers.

Corollary 3.5 Let $G$ be a linear group with polycyclic-by-finite layers. Then either $G$ is polycyclic-by-finite or it is an F L-group.

It is easy to see that a soluble group with layers of finite rank need not have in general finite rank itself, the group

$$
\underset{p \in \mathbb{P}}{\operatorname{Dr}}\left(C_{p} \times \ldots \times C_{p}\right)
$$

being a counterexample, where $\mathbb{P}$ is the set of all prime numbers and $C_{p}$ is a group of order $p$ for any prime $p$. Our final result shows that once again the situation is better for linear groups.

Theorem 3.6 Let $G$ be a linear group whose layers have finite rank. Then $G$ has finite rank.

Proof Let $S$ be the largest soluble normal subgroup of $G$. Then $S$ is closed, so $G / S$ is linear. Moreover, $G / S$ is covered by finite normal subgroups because every layer of $G$ is soluble-by-finite. Thus $G / S$ is $F C$, central-by-finite and so even finite. It follows that $G$ is soluble-by-finite and, without loss of generality, we may suppose that $G$ is soluble.

Let $A$ be any abelian subgroup of $G$. The assumption on the layers of $G$ yields that $A$ has finite $p$-rank for each $p=0$ or a prime. Therefore $G$ has finite abelian subgroup rank and so it has finite rank by a combination of results in [2] and Lemma 3.1 of [5].

Funding Open access funding provided by Universitá degli Studi di Napoli Federico II within the CRUICARE Agreement.

\section{Declarations}

Conflict of interest The authors state that there is no conflict of interest. 
Open Access This article is licensed under a Creative Commons Attribution 4.0 International License, which permits use, sharing, adaptation, distribution and reproduction in any medium or format, as long as you give appropriate credit to the original author(s) and the source, provide a link to the Creative Commons licence, and indicate if changes were made. The images or other third party material in this article are included in the article's Creative Commons licence, unless indicated otherwise in a credit line to the material. If material is not included in the article's Creative Commons licence and your intended use is not permitted by statutory regulation or exceeds the permitted use, you will need to obtain permission directly from the copyright holder. To view a copy of this licence, visit http://creativecommons.org/licenses/by/4.0/.

\section{References}

1. Baer, R.: Endlichkeitskriterien für Kommutatorgruppen. Math. Ann. 124, 161-177 (1952)

2. Baer, R., Heineken, H.: Radical groups of finite abelian subgroup rank. Ill. J. Math. 16, 533-580 (1972)

3. Franciosi, S., de Giovanni, F., Tomkinson, M.J.: Groups with polycyclic-by-finite conjugacy classes. Boll. Un. Mat. Ital. B 4, 35-55 (1990)

4. de Giovanni, F., Russo, A., Vincenzi, G.: Groups with restricted conjugacy classes. Serdica Math. J. 28, 241-254 (2002)

5. de Giovanni, F., Trombetti, M., Wehrfritz, B.A.F.: The upper and lower central series in linear groups. Q. J. Math. (to appear). https://doi.org/10.1093/qmath/haab030.

6. Hall, P.: Finite-by-nilpotent groups. Proc. Camb. Philos. Soc. 52, 611-616 (1956)

7. Kurdachenko, L.A.: Groups with minimax classes of conjugate elements. In: Infinite Groups and Related Algebraic Structures. Akad. Nauk Ukr. Inst. Mat. Kiev, pp 160-177 (1993)

8. Merzljakov, Yu.I.: Verbal and marginal subgroups of linear groups. Soviet Math. Dokl. 8, 1538-1541 (1967)

9. Polovickiı̌, Ya.D.: Groups with extremal classes of conjugate elements. Sibirsk. Mat. Ž. 5, 891-895 (1964)

10. Robinson, D.J.S.: Finiteness conditions and generalized soluble groups. Springer, Berlin (1972)

11. Schur, I.: Neuer Beweis eines Satzes über endliche Gruppen. In: Proceedings of symposia in applied mathematics, Sitzungsb. Preuss. Akad. Wiss., Berlin, pp 1013-1019 (1902)

12. Tomkinson, M.J.: FC-groups. Pitman, Boston (1984)

13. Wehrfritz, B.A.F.: Infinite linear groups. Springer, Berlin (1973)

Publisher's Note Springer Nature remains neutral with regard to jurisdictional claims in published maps and institutional affiliations. 